\title{
General Heat Kernel Coefficients for Massless Free Spin-3/2 Rarita-Schwinger Field
}

\author{
Sudip Karan, Shashank Kumar ${ }^{\dagger}$ and Binata Panda ${ }^{\ddagger}$ \\ Department of Applied Physics \\ Indian Institute of Technology (Indian School of Mines) \\ Dhanbad, Jharkhand-826004, INDIA
}

\begin{abstract}
We review the general heat kernel method for the Dirac spinor field as an elementary example in any arbitrary background. We, then compute the first three Seeley-DeWitt coefficients for the massless free spin-3/2 Rarita-Schwinger field without imposing any limitations on the background geometry.
\end{abstract}

* sudip.karaan@gmail.com

'shashankshimla23@gmail.com

binata@iitism.ac.in 


\section{Introduction}

Heat kernel is the fundamental solution to the heat equation for an elliptic partial differential operator on a specified domain with proper boundary conditions. It turns out that the heat kernel is one of the most important tools in spectral geometry, in differential geometry, and in global analysis more generally [1]. Moreover, asymptotics of the heat kernel are related to the eigenvalue asymptotics [2]. It is also a useful tool to study the index theorem of Atiyah and Singer [3,4].

Another motivation to study the heat kernel comes from physics. The important works by Fock [5] and Schwinger [6] introduced the heat kernel to quantum theory. Again, DeWitt's covariant approach [7-10] made heat kernel one of the powerful tools for quantum field theory and quantum gravity (also review [11 13]). The heat kernel expansion is a very convenient tool for studying one-loop divergences, anomalies, and various asymptotics of the effective action. In recent years, the progress in theoretical physics, especially in string theory and related areas made special use of heat kernel coefficients to calculate the logarithmic corrections to Bekenstein-Hawking black hole entropy [14 19]. These logarithmic corrections have great significance as they are aspects of the high energy theory, but one may compute them systematically in the low energy effective theory; which involves computation of heat kernel coefficient (more precisely coefficient $a_{4}$ introduced in eq. (2.9) ) for the kinetic differential operator of the massless fields in the black hole background.

In recent years, Rarita-Schwinger field has become one of the active research topics in theoretical physics. Although, Rarita-Schwinger field plays dominating role in supergravity, it has many other important applications. Analysis for massless Rarita-Schwinger field in covariant and the Coulomb-like gauges can be useful for perturbative interacting field theories [20]. Also, recent investigations [21-23] suggest that spin-3/2 may have a crucial role to play in explaining the evolution of the universe. It turns out that a detailed investigation of the heat kernel for the spin-3/2 Rarita-Schwinger field is useful for many research applications. These heat kernel coefficients for different fields were used to study quantum properties of different higher dimensional and dimensionally reduced supersymmetric theories [24,25]. The heat kernel coefficients for spin-3/2 field in general covariant gauge are expressed in terms of the heat kernel coefficients for spin- $1 / 2$ field following DeWitt's algorithm [26]. However, in the present paper, we are going to represent a general approach for calculating heat kernel coefficients for massless free spin-3/2 Rarita-Schwinger field in any arbitrary background. We start with reviewing the general heat kernel technique for some elementary examples of Dirac spinor field around an arbitrary background field configuration.

To illustrate the use of heat kernel in quantum field theory, let us consider the Gaussian form of the Euclidean path integral in one-loop approximation 1

$$
e^{-W}=\int[\mathcal{D} \phi] e^{-\phi \Lambda \phi}=\frac{1}{\sqrt{\operatorname{det} \Lambda}}
$$

where $\Lambda$ is a kinetic differential operator of background fields $\{\phi\}$ (schematic form is shown in eq. (2.3) ) and $W$ is the corresponding one-loop effective action. Also, one can express the one loop determinant $\operatorname{det} \Lambda$ as a product over the spectrum of $\Lambda$,

$$
\begin{gathered}
\operatorname{det} \Lambda=\prod_{i} \lambda_{i}, \\
W=\frac{1}{2} \ln \operatorname{det} \Lambda=\frac{1}{2} \sum_{i} \ln \lambda_{i} .
\end{gathered}
$$

We regularize the sum over eigenvalues $\left(\lambda_{i}^{\prime} s\right)$ using the heat kernel method. With the eigen-

\footnotetext{
${ }^{1}$ One loop approximation involves the expansion of the Lagrangian $\mathcal{L}(\Phi)$ of the system up to quadratic order in fluctuations $(\phi)$ around some classical background.
} 
functions of $\Lambda$ are given, one can modify the Green's function 2 as

$$
K\left(x, x^{\prime} ; s\right)=\sum_{i} e^{-s \lambda_{i}} f_{i}(x) f_{i}^{*}\left(x^{\prime}\right)
$$

which satisfies the heat equation

$$
\left(\partial_{s}+\Lambda_{x}\right) K\left(x, x^{\prime} ; s\right)=0
$$

hence the reference to heat. The heat kernel parameter $s$ has units of length squared. One can avoid the dependence on the eigenfunctions by setting $x=x^{\prime}$, integrating over the manifold of interest, and using the normalization of the eigenfunctions $f_{i}(x)$. The resulting function $D(s)$ will be referred as the heat kernel,

$$
D(s)=\int d^{4} x \sqrt{\operatorname{det} g_{\mu \nu}} K(x, x ; s)=\operatorname{tr}\left(e^{-s \Lambda}\right)=\sum_{i} e^{-s \lambda_{i}} .
$$

$D(s)$ is an object of interest for us because the one loop determinant (1.3) is then expressed as

$$
W=-\frac{1}{2} \int_{\epsilon^{2}}^{\infty} d s \frac{D(s)}{s},
$$

where $\epsilon$ is a UV regulator with the dimension of length, added by hand since the integral (1.7) diverges at small $s$. $W$ is important to our work as it is the input to Sen's quantum entropy function. Given the spectrum $\lambda_{i}$ of the kinetic operator $\Lambda$, one can evaluate the heat kernel using eq. (1.6). Once we get $D(s)$ in our hand, we can calculate the one loop effective action $W$ and thus one loop corrections to black hole entropy, given the knowledge of the spectrum of fields present around the black hole geometry.

The rest of this paper is organized as follows. In $\S 2$, we first review the general heat kernel method [13] for determining heat kernel coefficients for field fluctuations around any arbitrary background, and also list out general formulae for first three Seeley-DeWitt coefficients. Next, we employ this method to the different cases of Dirac spinor field as a warm up exercise. We consider the cases of free and gauged Dirac spinor fields for both massless and massive cases. Then, in $\S 3$ we compute Seeley-DeWitt coefficients for the spin-3/2 Rarita-Schwinger field following the same general approach and comment on the consistency of the results. In $\S$, we end by summarizing the results.

\section{General heat kernel method}

Let us start with a four-dimensional theory of gravity with an action having the form,

$$
\mathcal{S}=\int d^{4} x \sqrt{\operatorname{det} g_{\mu \nu}} \mathcal{L}
$$

where $g_{\mu \nu}$ is the background metrid 3 and $\mathcal{L}$ is the Lagrangian density. Considering upto quadratic order in fluctuations around a particular background, the action takes the schematic form,

$$
\mathcal{S}=\int d^{4} x \sqrt{\operatorname{det} g_{\mu \nu}} M^{m p} \phi_{p} \Lambda_{m}^{n} \phi_{n}
$$

where $M$ is some matrix, $\left\{\phi_{m}\right\}$ is the set of all the fluctuating fields, $\Lambda$ is the kinetic differential operator acting on quadratic field fluctuations.

\footnotetext{
${ }^{2}$ The Green's function is subject to the boundary condition $K\left(x, x^{\prime} ; s=0\right)=\delta\left(x-x^{\prime}\right)$, a consequence of the orthonormality of the eigenfunctions $f_{i}(x)$.

${ }^{3}$ The Euclidean signature of the metric is assumed in this paper; in our convention $\operatorname{sig}\left(g_{\mu \nu}\right)=(++++)$.
} 
The present method demands $\Lambda$ to be both Hermitian and Laplace-type. To make $\Lambda$ as Hermitian, we must restructure our action (up to a total derivative) while for the later requirement, we choose $\Lambda$ to have the following Laplace-type form

$$
\Lambda_{m}^{n}=-\left(D^{\mu} D_{\mu}\right) I_{m}^{n}-\left(N^{\mu} D_{\mu}\right)_{m}^{n}-P_{m}^{n}
$$

where $D_{\mu}$ is the ordinary covariant derivative with connections determined by the background metric; $N$ and $P$ are arbitrary matrices constructed from the background fields; and $I$ is the corresponding identity operator on the space of fields.

Using the definition $\mathcal{D}_{\mu}=D_{\mu}+\omega_{\mu}$ in eq. (2.3) we can rewrite $\Lambda$ as

$$
\Lambda_{m}^{n}=-\left(\mathcal{D}^{\mu} \mathcal{D}_{\mu}\right) I_{m}^{n}-\left\{P_{m}^{n}-\left(\omega^{\mu} \omega_{\mu}\right)_{m}^{n}-\left(D^{\mu} \omega_{\mu}\right)_{m}^{n}\right\}-\left\{\left(N^{\mu}-2 \omega^{\mu}\right) D_{\mu}\right\}_{m}^{n} .
$$

Finally, one can compare eq. (2.4) with the standard form

$$
\Lambda=-\left(\mathcal{D}^{\mu} \mathcal{D}_{\mu}\right) I-E,
$$

and identify the following useful matrices

$$
\begin{gathered}
\omega_{\mu}=\frac{1}{2} N_{\mu}, \\
E=P-\left(\omega^{\mu} \omega_{\mu}\right)-\left(D^{\mu} \omega_{\mu}\right) .
\end{gathered}
$$

Note that the term in parentheses $\left(D^{\mu} \omega_{\mu}\right)$ indicates the covariant derivative $D^{\mu}$ acting only on $\omega_{\mu}$. The curvature associated with $\mathcal{D}_{\mu}$ is defined as

$$
\begin{aligned}
\Omega_{\mu \nu} & \equiv\left[\mathcal{D}_{\mu}, \mathcal{D}_{\nu}\right] \\
& =\left[D_{\mu}, D_{\nu}\right]+D_{[\mu} \omega_{\nu]}+\left[\omega_{\mu}, \omega_{\nu}\right] .
\end{aligned}
$$

\subsection{General formulae}

The heat kernel corresponding to the differential operator $\Lambda$ is defined in eq. (1.6), and for small $s$ its perturbative expansion has the following form [7-10, 13, 27, 35]

$$
\begin{aligned}
D(s) & \cong \int d^{4} x \sqrt{\operatorname{det} g_{\mu \nu}} \sum_{n=0}^{\infty} s^{n-2} a_{2 n}(x) \\
& =\int d^{4} x \sqrt{\operatorname{det} g_{\mu \nu}}\left\{\frac{1}{s^{2}} a_{0}(x)+\frac{1}{s} a_{2}(x)+a_{4}(x)+\cdots\right\},
\end{aligned}
$$

where the coefficients $a_{2 n}(x)$ are known as Seeley-DeWitt coefficients4 [7, 10, 27, 28]. We want to express these coefficients in terms of local invariants obtained from background metric $\left(g_{\mu \nu}\right)$, Riemann tensor $\left(R_{\mu \nu \rho \sigma}\right)$, Ricci tensor $\left(R_{\mu \nu}\right)$, Ricci scalar $(R)$, gauge field strength $\left(F_{\mu \nu}\right)$ and their covariant derivatives. Once computed we can employ this general result for any arbitrary background field configuration.

With the definitions discussed in this section, the results for the leading Seeley-DeWitt coefficients (see eqs. 4.26, 4.27 and 4.28 of [13]) are summarized in the following equations 5

$$
\begin{gathered}
(4 \pi)^{2} a_{0}(x)=\operatorname{tr}(I), \\
(4 \pi)^{2} a_{2}(x)=\frac{1}{6} \operatorname{tr}(6 E+R I), \\
(4 \pi)^{2} a_{4}(x)=\frac{1}{360} \operatorname{tr}\left\{60 R E+180 E^{2}+30 \Omega_{\mu \nu} \Omega^{\mu \nu}+\left(5 R^{2}+2 R_{\mu \nu \rho \sigma} R^{\mu \nu \rho \sigma}-2 R_{\mu \nu} R^{\mu \nu}\right) I\right\} .
\end{gathered}
$$

\footnotetext{
${ }^{4}$ In eq. (2.8), the terms $\int d^{4} x \sqrt{\operatorname{det} g_{\mu \nu}} a_{2 n}(x)$ are our desired heat kernel coefficients and to obtain these coefficients, we must choose a particular manifold to perform the integration. But our aim is to calculate generalized form heat kernel coefficients for spin-3/2 Rarita-Schwinger field. So, we focus on computation of Seeley-DeWitt coefficients instead.

${ }^{5}$ The formulae given in eq. (2.9) are only valid for manifolds without boundary.
} 
We note that all the total derivative terms are neglected as they will serve as boundary terms in the integral eq. (2.8) and hence will vanish after integration. Here, "tr" denotes trace over the field index $m$. One can evaluate curvature tensors and curvature scalar from the background metric $\left(g_{\mu \nu}\right)$ and the results for $E, I$ and $\Omega$ can be obtained from eqs. (2.6) and (2.7). In principle, we can compute higher order Seeley-DeWitt coefficients, $a_{6}(x), a_{8}(x), \cdots$ as per requirements in this method.

\subsection{Elementary examples}

In this section, we start with illustrating the general heat kernel approach to calculate the heat kernel coefficients for Dirac spinor field as an elementary example. We calculate first three SeeleyDeWitt coefficients for four different cases namely massless free Dirac spinor field, massive free Dirac spinor field, massless gauged Dirac spinor field, and massive gauged Dirac spinor field.

\subsubsection{Free Dirac spinor field}

The classical Lagrangian for a massless free Dirac spinor $\psi$ is given by

$$
\mathcal{L}=i \bar{\psi} \gamma^{\mu} D_{\mu} \psi
$$

where $D_{\mu}$ is the covariant derivative acting on the spinors and the gamma matrices $\left(\gamma^{\mu}\right)$ satisfy the standard 4D Clifford algebra,

$$
\gamma^{\mu} \gamma^{\nu}+\gamma^{\nu} \gamma^{\mu}=2 g^{\mu \nu} \mathbb{I}_{4}
$$

with $\mathbb{I}_{4}$ being the identity matrix in Clifford algebra. The Lagrangian (2.10) contains firstorder Dirac-type differential operator $\not D=i \gamma^{\mu} D_{\mu}$, but our aim is to make it quadratic in order. Assuming our spacetime to be even-dimensional having Euclidean signature, our gamma matrices are Hermitian, $\gamma_{\mu}^{\dagger}=\gamma_{\mu}$ and hence the operator $\gamma^{\mu} D_{\mu}$ is anti-Hermitian, $\left(\gamma^{\mu} D_{\mu}\right)^{\dagger}=-\gamma^{\mu} D_{\mu}$. With this choice, the differential operator $\not D$ is Hermitian.

We then compute the relevant second-order Laplace-type differential operator 6 acting on $\psi$,

$$
\begin{aligned}
\Lambda=\not D^{2} & =i^{2} \gamma^{\mu} \gamma^{\nu} D_{\mu} D_{\nu} \\
& =-\left(D^{\mu} D_{\mu}\right)-\frac{1}{2} \gamma^{\mu} \gamma^{\nu}\left[D_{\mu}, D_{\nu}\right]
\end{aligned}
$$

Gamma matrix commutation relation gives,

$$
\left[D_{\mu}, D_{\nu}\right] \psi=\frac{1}{4} \gamma^{\theta} \gamma^{\phi} R_{\mu \nu \theta \phi} \psi
$$

Also, using gamma identity (2.11) and standard Riemann identities, we can express

$$
\gamma^{\mu} \gamma^{\nu} \gamma^{\theta} \gamma^{\phi} R_{\mu \nu \theta \phi}=-2 R
$$

Inserting relations (2.13) and (2.14) in eq.(2.12), we find that

$$
\bar{\psi} \Lambda \psi=-\bar{\psi}\left(D^{\mu} D_{\mu}\right) \psi+\frac{R}{4} \bar{\psi} \psi
$$

Finally, we can rewrite eq.(2.15) using the definition $\mathcal{D}_{\mu}=D_{\mu}+\omega_{\mu}$,

$$
\bar{\psi} \Lambda \psi=\bar{\psi}\left\{-\mathcal{D}^{\mu} \mathcal{D}_{\mu}+D^{\mu} \omega_{\mu}+\omega_{\mu} \omega^{\mu}+\frac{R}{4}+2 \omega^{\mu} D_{\mu}\right\} \psi
$$

\footnotetext{
${ }^{6}$ With our choice of four-dimensional space-time, $\not D$ is Hermitian and we can express $\Lambda=\not D^{\dagger} \not D=\not D D^{2}$.
} 
The form of $\Lambda$ given in eq. (2.16) is of the Laplace-type form as required in eq. (2.4) and one can compare this $\Lambda$ with eq. (2.5) and easily extract following useful matrices with the help of eq. (2.6)

$$
I=\mathbb{I}_{4}, \quad \omega_{\mu}=0, \quad E=-\frac{R}{4} \mathbb{I}_{4}, \quad \Omega_{\mu \nu}=\frac{1}{4} \gamma^{\theta} \gamma^{\phi} R_{\mu \nu \theta \phi},
$$

where in calculating $\Omega_{\mu \nu}$ we have used eq. (2.13) in the definition of $\Omega$ given in eq. (2.7).

Next, we will use gamma contraction identities [36] and compute all the necessary traces which are useful for obtaining Seeley-DeWitt coefficients,

$$
\operatorname{tr}(I)=4, \operatorname{tr}(E)=-R, \operatorname{tr}\left(E^{2}\right)=\frac{R^{2}}{4}, \operatorname{tr}\left(\Omega_{\mu \nu} \Omega^{\mu \nu}\right)=-\frac{1}{2} R_{\mu \nu \rho \sigma} R^{\mu \nu \rho \sigma} .
$$

Inserting these data into eq. (2.9), we find Seeley-DeWitt coefficients for massless free Dirac spinor field as

$$
\begin{gathered}
(4 \pi)^{2} a_{0}(x)=-4, \\
(4 \pi)^{2} a_{2}(x)=+\frac{R}{3}, \\
(4 \pi)^{2} a_{4}(x)=-\frac{1}{360}\left(5 R^{2}-8 R_{\mu \nu} R^{\mu \nu}-7 R_{\mu \nu \rho \sigma} R^{\mu \nu \rho \sigma}\right) .
\end{gathered}
$$

Note that we have put an overall minus sign by hand on each Seeley-DeWitt coefficients to account for fermion statistics [37] and we will do the same for all the spinor cases.

Our next aim is to study the case of massive free Dirac spinor (with real mass $m$ ) and for that, we have to add a mass term in the Lagrangian given in eq. (2.10),

$$
\mathcal{L}=\bar{\psi}\left\{i \gamma^{\mu} D_{\mu}+m\right\} \psi
$$

and the corresponding Laplace-type operator can be obtained by following the technique discussed in [37],

$$
\begin{aligned}
\Lambda & =\left(i \gamma^{\mu} D_{\mu}+m\right) \times\left(i \gamma^{\nu} D_{\nu}-m\right) \\
& =-\gamma^{\mu} \gamma^{\nu} D_{\mu} D_{\nu}-m^{2} .
\end{aligned}
$$

Proceeding in the same way as for massless Dirac spinor field, we obtain

$$
\begin{gathered}
E=\left(m^{2}-\frac{R}{4}\right) \mathbb{I}_{4}, \\
\operatorname{tr}(E)=4 m^{2}-R \\
\operatorname{tr}\left(E^{2}\right)=4 m^{4}-2 m^{2} R+\frac{R^{2}}{4},
\end{gathered}
$$

while other matrices like $I, \omega, \Omega$ and their traces remain exactly same as in eqs. (2.17) and (2.18).

Using the results in eq. (2.9), we obtain the Seeley-DeWitt coefficients for massive free Dirac spinor field as

$$
\begin{gathered}
(4 \pi)^{2} a_{0}(x)=-4, \\
(4 \pi)^{2} a_{2}(x)=-\left(4 m^{2}-\frac{R}{3}\right), \\
(4 \pi)^{2} a_{4}(x)=-\frac{1}{360}\left(720 m^{4}-120 m^{2} R+5 R^{2}-8 R_{\mu \nu} R^{\mu \nu}-7 R_{\mu \nu \rho \sigma} R^{\mu \nu \rho \sigma}\right) .
\end{gathered}
$$




\subsubsection{Dirac spinor field with gauge connection}

The standard form of Lagrangian for a massless Dirac spinor field $\psi$ with gauge field connection $A_{\mu}$ is given by

$$
\mathcal{L}=i \bar{\psi} \gamma^{\mu}\left(D_{\mu}+A_{\mu}\right) \psi
$$

With our choice of space-time which is even dimensional and has euclidean signature, $A_{\mu}$ is antiHermitian in the gauge indices, $A_{\mu}^{\dagger}=-A_{\mu}$; also, $\gamma^{\mu} D_{\mu}$ is anti-Hermitian, $\left(\gamma^{\mu} D_{\mu}\right)^{\dagger}=-\gamma^{\mu} D_{\mu}$; and hence, $\not D=i \gamma^{\mu}\left(D_{\mu}+A_{\mu}\right)$ is Hermitian. We employ the same approach as we did in $\S 2.2 .1$ and find that the relevant second-order differential operator acting on $\psi$ takes the following form

$$
\begin{aligned}
\Lambda=\not D^{2} & =i \gamma^{\mu}\left(D_{\mu}+A_{\mu}\right) \times i \gamma^{\nu}\left(D_{\nu}+A_{\nu}\right) \\
& =-D^{\mu} D_{\mu}-\frac{1}{2} \gamma^{\mu} \gamma^{\nu}\left[D_{\mu}, D_{\nu}\right]-\frac{1}{2} \gamma^{\mu} \gamma^{\nu} F_{\mu \nu}-D^{\mu} A_{\mu}-2 A^{\mu} D_{\mu}-A^{\mu} A_{\mu}
\end{aligned}
$$

where the notation $F_{\mu \nu}=\partial_{\mu} A_{\nu}-\partial_{\nu} A_{\mu}+\left[A_{\mu}, A_{\nu}\right]$.

Now, using the result of eq. (2.16) we find for massless gauged spinor field,

$$
\bar{\psi} \Lambda \psi=\bar{\psi}\left\{-\mathcal{D}^{\mu} \mathcal{D}_{\mu}+D^{\mu} \omega_{\mu}+\omega^{\mu} \omega_{\mu}-\frac{1}{2} \gamma^{\mu} \gamma^{\nu} F_{\mu \nu}-D^{\mu} A_{\mu}-A^{\mu} A_{\mu}+\frac{R}{4}+2\left(\omega^{\mu}-A^{\mu}\right) D_{\mu}\right\} \bar{\psi}
$$

Finally, comparing the form of $\Lambda$ from eq. (2.26) with eq. (2.5) and using eq. (2.6), we identify $I, \omega_{\mu}, E$, and $\Omega_{\mu \nu}$ as

$$
\begin{aligned}
I & =\mathbb{I}_{4}, \omega_{\mu}=A_{\mu}, \\
E & =-D^{\mu} \omega_{\mu}-\omega_{\mu} \omega^{\mu}+\frac{1}{2} \gamma^{\mu} \gamma^{\nu} F_{\mu \nu}+D^{\mu} A_{\mu}+A^{\mu} A_{\mu}-\frac{R}{4} \mathbb{I}_{4} \\
& =\frac{1}{2} \gamma^{\mu} \gamma^{\nu} F_{\mu \nu}-\frac{R}{4} \mathbb{I}_{4}, \\
\Omega_{\mu \nu} & =\left[D_{\mu}, D_{\nu}\right]+D_{[\mu} \omega_{\nu]}+\left[\omega_{\mu}, \omega_{\nu}\right] \\
& =\frac{1}{4} \gamma^{\theta} \gamma^{\phi} R_{\mu \nu \theta \phi}+F_{\mu \nu},
\end{aligned}
$$

Note that, for obtaining $\Omega$, we have substituted the relation, (2.13) in the definition (2.7).

Now, our task is to calculate all the traces necessary for calculating Seeley-DeWitt coefficients. The results are

$$
\begin{aligned}
\operatorname{tr}(I) & =4 \\
\operatorname{tr}(E) & =-R, \\
\operatorname{tr}\left(E^{2}\right) & =\frac{R^{2}}{4}-2 F_{\mu \nu} F^{\mu \nu}, \\
\operatorname{tr}\left(\Omega_{\mu \nu} \Omega^{\mu \nu}\right) & =4 F_{\mu \nu} F^{\mu \nu}-\frac{1}{2} R_{\mu \nu \rho \sigma} R^{\mu \nu \rho \sigma} .
\end{aligned}
$$

Here, we have used standard gamma contraction identities [36] and Riemann identities for the necessary simplifications.

We use the result of these traces in the formulae (2.9) to calculate the Seeley-DeWitt coefficients for the massless gauged Dirac spinor field,

$$
\begin{gathered}
(4 \pi)^{2} a_{0}(x)=-4 \\
(4 \pi)^{2} a_{2}(x)=+\frac{R}{3} \\
(4 \pi)^{2} a_{4}(x)=-\frac{1}{360}\left(5 R^{2}-8 R_{\mu \nu} R^{\mu \nu}-7 R_{\mu \nu \rho \sigma} R^{\mu \nu \rho \sigma}-240 F_{\mu \nu} F^{\mu \nu}\right) .
\end{gathered}
$$


Next, we are going to study the case of massive gauged Dirac spinor field having the following form of Lagrangian

$$
\mathcal{L}=\bar{\psi}\left\{i \gamma^{\mu}\left(D_{\mu}+A_{\mu}\right)+m\right\} \psi .
$$

To deal with the mass term, we use the same technique as we did for massive free Dirac spinor in $\S 2.2 .1$ and find the required second-order differential operator as

$$
\begin{aligned}
\Lambda & =\left\{i \gamma^{\mu}\left(D_{\mu}+A_{\mu}\right)+m\right\} \times\left\{i \gamma^{\nu}\left(D_{\nu}+A_{\nu}\right)-m\right\} \\
& =-\gamma^{\mu} \gamma^{\nu}\left(D_{\mu}+A_{\mu}\right)\left(D_{\nu}+A_{\nu}\right)-m^{2} .
\end{aligned}
$$

Using the results from eqs. (2.25) and (2.26), we obtain the necessary matrices and traces as

$$
\begin{aligned}
E & =\frac{1}{2} \gamma^{\mu} \gamma^{\nu} F_{\mu \nu}+\left(m^{2}-\frac{R}{4}\right) \mathbb{I}_{4}, \\
\operatorname{tr}(E) & =4 m^{2}-R \\
\operatorname{tr}\left(E^{2}\right) & =4 m^{4}-2 m^{2} R+\frac{R^{2}}{4}-2 F_{\mu \nu} F^{\mu \nu},
\end{aligned}
$$

while other matrices $I, \omega$ and $\Omega$, and their traces remain exactly the same as in eqs. (2.27) and (2.28).

Now, substituting this data in the formulae (2.9), we obtain Seeley-DeWitt coefficients for the massive gauged Dirac spinor field as

$$
\begin{gathered}
(4 \pi)^{2} a_{0}(x)=-4, \\
(4 \pi)^{2} a_{2}(x)=-\left(4 m^{2}-\frac{R}{3}\right), \\
(4 \pi)^{2} a_{4}(x)=-\frac{1}{360}\left(720 m^{4}-120 m^{2} R+5 R^{2}-8 R_{\mu \nu} R^{\mu \nu}-7 R_{\mu \nu \rho \sigma} R^{\mu \nu \rho \sigma}-240 F_{\mu \nu} F^{\mu \nu}\right) .
\end{gathered}
$$

\section{Spin-3/2 Rarita-Schwinger field}

The spin-3/2 particle theory was first proposed by W. Rarita and J. Schwinger [38], based on Fierz and Pauli construction of field theory with arbitrary spins. In four-dimensional space-time, the Lagrangian of a massless free RS field $\psi_{\mu}$ is given by

$$
\mathcal{L}_{R S}=-i \bar{\psi}_{\mu} \gamma^{\mu \lambda \nu} D_{\lambda} \psi_{\nu}
$$

where $\gamma^{\mu \lambda \nu}$ is the antisymmetrized product of gamma matrices with respect to the indices $\mu, \lambda$, and $\nu$. Using the relation (2.11), one can simplify $\gamma^{\mu \lambda \nu}$ and rewrite $\mathcal{L}_{R S}$ as

$$
\mathcal{L}_{R S}=-\frac{i}{2} \bar{\psi}_{\mu}\left(\gamma^{\mu} \gamma^{\lambda} \gamma^{\nu}-\gamma^{\nu} \gamma^{\lambda} \gamma^{\mu}\right) D_{\lambda} \psi_{\nu}
$$

We gauge fix the theory by adding the following gauge fixing term to the Lagrangian in eq. (3.2)

$$
\mathcal{L}_{G F}=\frac{i}{2} \bar{\psi}_{\mu} \gamma^{\mu} \gamma^{\lambda} \gamma^{\nu} D_{\lambda} \psi_{\nu}
$$

As a result, the total gauge fixed Lagrangian takes the following form

$$
\mathcal{L}=\mathcal{L}_{R S}+\mathcal{L}_{G F}=\frac{i}{2} \bar{\psi}_{\mu} \gamma^{\nu} \gamma^{\lambda} \gamma^{\mu} D_{\lambda} \psi_{\nu}
$$

The Lagrangian consists of a first-order Dirac-type differential operator $\not D^{\mu \nu} \equiv \frac{i}{2} \gamma^{\nu} \gamma^{\lambda} \gamma^{\mu} D_{\lambda}$ and with our choice of space-time, $\not D$ is Hermitian. In order to obtain the corresponding second-order 
Laplace-type operator, let's consider

$$
\begin{aligned}
\Lambda^{\mu \nu}=\left(\not D^{2}\right)^{\mu \nu} & =\not D^{\mu \sigma} \not D_{\sigma}^{\nu} \\
& =\left(\frac{i}{2} \gamma^{\sigma} \gamma^{\rho} \gamma^{\mu} D_{\rho}\right) \times\left(\frac{i}{2} \gamma^{\nu} \gamma^{\lambda} \gamma_{\sigma} D_{\lambda}\right) \\
& =-\frac{1}{2}\left(\gamma^{\lambda} \gamma^{\rho} \gamma^{\mu} \gamma^{\nu}+\gamma^{\nu} \gamma^{\mu} \gamma^{\rho} \gamma^{\lambda}\right) D_{\rho} D_{\lambda} \\
& =-g^{\mu \nu}\left(D^{\rho} D_{\rho}\right)+\frac{1}{4}\left(\gamma^{\rho} \gamma^{\lambda} \gamma^{\mu} \gamma^{\nu}+\gamma^{\nu} \gamma^{\mu} \gamma^{\lambda} \gamma^{\rho}\right)\left[D_{\rho}, D_{\lambda}\right]
\end{aligned}
$$

where equality in the third line is obtained using the gamma matrix contraction identity [36]. In order to simplify the expression eq. (3.5), we now consider

$$
\bar{\psi}_{\mu} \Lambda^{\mu \nu} \psi_{\nu}=\bar{\psi}_{\mu}\left\{-g^{\mu \nu} D^{\rho} D_{\rho}+\frac{1}{4}\left(\gamma^{\rho} \gamma^{\lambda} \gamma^{\mu} \gamma^{\nu}+\gamma^{\nu} \gamma^{\mu} \gamma^{\lambda} \gamma^{\rho}\right)\left[D_{\rho}, D_{\lambda}\right]\right\} \psi_{\nu}
$$

Now, one can proceed in a systematic manner and simplify eq. (3.6) using the following identities

$$
\begin{gathered}
{\left[D_{\rho}, D_{\lambda}\right] \psi_{\nu}=-R_{\nu \rho \lambda}^{\sigma} \psi_{\sigma}+\frac{1}{4} R_{\rho \lambda \theta \phi} \gamma^{\theta} \gamma^{\phi} \psi_{\nu}} \\
\left(\gamma^{\rho} \gamma^{\lambda} \gamma^{\mu} \gamma^{\sigma}+\gamma^{\sigma} \gamma^{\mu} \gamma^{\lambda} \gamma^{\rho}\right) R^{\nu}{ }_{\sigma \rho \lambda}=-4 R^{\mu \nu}, \\
\left(\gamma^{\rho} \gamma^{\lambda} \gamma^{\mu} \gamma^{\nu}+\gamma^{\nu} \gamma^{\mu} \gamma^{\lambda} \gamma^{\rho}\right) R_{\rho \lambda \theta \phi} \gamma^{\theta} \gamma^{\phi}=8\left(-\gamma^{\rho} \gamma^{\theta} R_{\rho \theta}^{\mu \nu}+\gamma^{\mu} \gamma^{\theta} R_{\theta}^{\nu}-\gamma^{\nu} \gamma^{\theta} R^{\mu}{ }_{\theta}\right) \\
-4 R\left(\gamma^{\mu} \gamma^{\nu}-g^{\mu \nu}\right),
\end{gathered}
$$

to get the final form of Laplace-type differential operator as

$$
\Lambda^{\mu \nu}=-g^{\mu \nu} \mathcal{D}^{\rho} \mathcal{D}_{\rho}+R^{\mu \nu}-\frac{1}{2} \gamma^{\rho} \gamma^{\theta} R_{\rho \theta}^{\mu \nu}+\frac{1}{2} \gamma^{\mu} \gamma^{\theta} R_{\theta}^{\nu}-\frac{1}{2} \gamma^{\nu} \gamma^{\theta} R_{\theta}^{\mu}-\frac{R}{4} \gamma^{\mu} \gamma^{\nu}+\frac{R}{4} g^{\mu \nu} .
$$

Note that, we have set $\omega=0$ in the following definition as $\omega$ vanishes for any free field,

$$
\mathcal{D}_{\alpha} \psi_{\mu}=D_{\alpha} \psi_{\mu}+\left(\omega_{\alpha}\right)_{\mu}{ }^{\nu} \psi_{\nu} .
$$

Comparing $\Lambda$ from eq. (3.8) with eq. (2.5), we identify $I, E$ and $\Omega_{\mu \nu}$ as

$$
\begin{aligned}
I^{\mu \nu} & =\mathbb{I}_{4} g^{\mu \nu}, \\
E^{\mu \nu} & =-\mathbb{I}_{4} R^{\mu \nu}+\frac{1}{2} \gamma^{\rho} \gamma^{\theta} R^{\mu \nu}{ }_{\rho \theta}-\frac{1}{2} \gamma^{\mu} \gamma^{\theta} R^{\nu}{ }_{\theta}+\frac{1}{2} \gamma^{\nu} \gamma^{\theta} R^{\mu}{ }_{\theta}+\frac{R}{4} \gamma^{\mu} \gamma^{\nu}-\frac{R}{4} \mathbb{I}_{4} g^{\mu \nu}, \\
\left(\Omega_{\mu \nu}\right)^{\alpha \beta} & =\mathbb{I}_{4} R^{\alpha \beta}{ }_{\mu \nu}+\frac{1}{4} g^{\alpha \beta} R_{\mu \nu \rho \lambda} \gamma^{\rho} \gamma^{\lambda},
\end{aligned}
$$

where we have used eqs. (3.7) and (3.9) in the definition of $\Omega$ given in eq. (2.7) to obtain its present form.

\subsection{Trace calculations}

Now, we compute the traces necessary for evaluation of Seeley-DeWitt coefficients using the matrices (3.10). Note that for this purpose, we will make use of Riemann identities, and the necessary gamma trace and contraction identities [36]. The trace results are as follows:

$$
\begin{gathered}
\operatorname{tr}(I)=16 . \\
\operatorname{tr}(E)=g_{\mu \nu}\left\{-\mathbb{I}_{4} R^{\mu \nu}+\frac{1}{2} \gamma^{\rho} \gamma^{\theta} R^{\mu \nu}{ }_{\rho \theta}-\frac{1}{2} \gamma^{\mu} \gamma^{\theta} R^{\nu}{ }_{\theta}+\frac{1}{2} \gamma^{\nu} \gamma^{\theta} R^{\mu}{ }_{\theta}+\frac{R}{4} \gamma^{\mu} \gamma^{\nu}-\frac{R}{4} \mathbb{I}_{4} g^{\mu \nu}\right\} \\
=-4 R,
\end{gathered}
$$




$$
\begin{aligned}
\operatorname{tr}\left(E^{2}\right)= & (\underbrace{-\mathbb{I}_{4} R^{\mu \nu}}_{A}+\overbrace{\frac{1}{2} \gamma^{\rho} \gamma^{\theta} R^{\mu \nu}{ }_{\rho \theta}}^{B} \underbrace{-\frac{1}{2} \gamma^{\mu} \gamma^{\theta} R^{\nu}{ }_{\theta}}_{C}+\overbrace{\frac{1}{2} \gamma^{\nu} \gamma^{\theta} R^{\mu}{ }_{\theta} \underbrace{+\frac{R}{4} \gamma^{\mu} \gamma^{\nu}}-\overbrace{F}^{\frac{R}{4} \mathbb{I}_{4} g^{\mu \nu}}}^{G}) \\
& \times(\overbrace{-\mathbb{I}_{4} R_{\nu \mu}}^{B} \underbrace{+\frac{1}{2} \gamma^{\sigma} \gamma^{\phi} R_{\nu \mu \sigma \phi}}_{2} \overbrace{-\frac{1}{2} \gamma_{\nu} \gamma^{\phi} R_{\mu \phi}}^{B}+\underbrace{\frac{1}{2} \gamma_{\mu} \gamma^{\phi} R_{\nu \phi}}_{4}+\overbrace{\frac{R}{4} \gamma_{\nu} \gamma_{\mu}}^{5} \underbrace{-\frac{R}{4} \mathbb{I}_{4} g_{\nu \mu}}_{6}) .
\end{aligned}
$$

Note that each term in eq. (3.13) is denoted by a corresponding symbol, and now we are going compute their products separately as follows

$$
\begin{aligned}
& A 2=B 1=0 \quad B 6=G 2=0 \\
& A 1=4 R_{\mu \nu} R^{\mu \nu} \\
& A 3=C 1=2 R_{\mu \nu} R^{\mu \nu} \\
& C 4=D 3=2 R_{\mu \nu} R^{\mu \nu} \\
& B 2=2 R_{\mu \nu \rho \sigma} R^{\mu \nu \rho \sigma} \\
& A 4=D 1=-2 R_{\mu \nu} R^{\mu \nu} \\
& C 5=F 3=-2 R^{2} \\
& C 3=R^{2} \\
& A 5=F 1=-R^{2} \\
& D 4=R^{2} \\
& A 6=G 1=R^{2} \\
& F 5=4 R^{2} \\
& B 3=C 2=-2 R_{\mu \nu} R^{\mu \nu} \\
& G 6=R^{2} \\
& B 4=D 2=-2 R_{\mu \nu} R^{\mu \nu} \\
& C 6=G 3=\frac{1}{2} R^{2} \\
& D 5=F 4=-R^{2} \\
& D 6=G 4=-\frac{1}{2} R^{2} \\
& B 5=F 2=R^{2} \\
& F 6=G 5=-R^{2}
\end{aligned}
$$

One may use the results of these terms in eq. (3.13) and finally can come up with the following result

$$
\operatorname{tr}\left(E^{2}\right)=2 R_{\mu \nu \rho \sigma} R^{\mu \nu \rho \sigma}+R^{2} .
$$

We will also use the result for $\Omega$ from eq. (3.10) and evaluate

$$
\begin{aligned}
\operatorname{tr}\left(\Omega_{\mu \nu} \Omega^{\mu \nu}\right) & =\left\{\mathbb{I}_{4} R_{\mu \nu}^{\alpha \beta}+\frac{1}{4} g^{\alpha \beta} \gamma^{\rho} \gamma^{\lambda} R_{\mu \nu \rho \lambda}\right\} \times\left\{\mathbb{I}_{4} R_{\beta \alpha}{ }^{\mu \nu}+\frac{1}{4} g_{\beta \alpha} \gamma^{\theta} \gamma^{\phi} R_{\mu \nu \theta \phi}\right\} \\
& =-6 R_{\mu \nu \rho \sigma} R^{\mu \nu \rho \sigma} .
\end{aligned}
$$

\subsection{Seeley-DeWitt coefficients}

One can substitute all the traces calculated in $\S 3.1$ in the standard formulae (2.9) in order to find the leading Seeley-DeWitt coefficients for the case of massless free spin-3/2 Rarita-Schwinger fermions. The results are

$$
\begin{gathered}
(4 \pi)^{2} a_{0}(x)=-16, \\
(4 \pi)^{2} a_{2}(x)=+\frac{4}{3} R, \\
(4 \pi)^{2} a_{4}(x)=-\frac{1}{360}\left(212 R_{\mu \nu \rho \sigma} R^{\mu \nu \rho \sigma}-32 R_{\mu \nu} R^{\mu \nu}+20 R^{2}\right) .
\end{gathered}
$$

For the background satisfying the condition $R=0$, one has to ignore the terms proportional to $\mathrm{R}$ in eq. (3.16). In case of Einstein manifold, one can arrive at the following results by setting $R_{\mu \nu}=\lambda g_{\mu \nu}(\lambda$ is arbitrary constant) in eq. (3.16)

$$
\begin{gathered}
(4 \pi)^{2} a_{0}(x)=-16, \\
(4 \pi)^{2} a_{2}(x)=+\frac{16}{3} \lambda, \\
(4 \pi)^{2} a_{4}(x)=-\frac{1}{360}\left(212 R_{\mu \nu \rho \sigma} R^{\mu \nu \rho \sigma}+192 \lambda^{2}\right) .
\end{gathered}
$$


Setting $\lambda=0$ in eq. (3.17), will provide the results for the Ricci-flat space-time $\left(R_{\mu \nu}=0\right)$. Note that, here also we have placed an overall minus sign by hand on each coefficient to account for fermion statistics.

The above results are clearly consistent with ref. [14] for $R=0$ manifold, where the authors have calculated the Seeley-DeWitt coefficients for fermions in $\mathcal{N}=2$ gravity multiplet 7 . One can extract the results for single free gravitino field, by ignoring the field-type indices $A, B$ and setting $F_{\mu \nu}=0$ in eqs 3.79 and 3.80 of ref. [14]. Also, for the special case of Ricci-flat manifold one can check the consistency from ref. [26]. Considering the result for $d=4$ and performing appropriate traces over eq. 24 of ref. [26], one can obtain the Seeley-DeWitt coefficients which are identical with our result (3.17) for $\lambda=0$.

\section{Summary}

In this report, we have computed the first three Seeley-DeWitt coefficients for the massless free spin-3/2 field and expressed them in terms of the local invariants of the theory. We started with the discussion of the general heat kernel approach and worked out a practice calculation for Seeley-DeWitt coefficients for spin-1/2 field as an elementary example. Then, we applied the same approach to calculate the Seeley-DeWitt coefficients for spin-3/2 Rarita-Schwinger field and discussed about the consistency of our results for various special cases. Apart from various interests, spin-3/2 Rarita-Schwinger field has a particular role in supergravity and evolution of the universe. However, we are interested in computing the heat kernel coefficients for the same in any arbitrary background as these coefficients have various applications in quantum gravity, quantum field theory, and black hole entropy corrections (mainly logarithmic corrections). One can employ these results of Seeley-DeWitt coefficients to compute the heat kernel coefficients by integrating them over the manifold of interest as discussed in $\S$ 2.1. We also note that the derivation in this paper assumes that $\psi$ is a Dirac spinor. Anyone interested in computing the heat kernel coefficients for Majorana or Weyl fermions instead of Dirac fermions can simply divide the results by two, since Weyl and Majorana spinors have half the degrees of freedom of Dirac spinor.

\section{Acknowledgments}

We would like to thank Gourav Banerjee for useful discussions. B.P. acknowledges IIT(ISM), Dhanbad for the Grant (FRS (53)/2013-2014/APH).

\section{References}

[1] P. Gilkey, Invariance Theory, the Heat Equation, and the Atiya-SingerIndex Theorem, CRC Press, Boca Raton, FL, (1995).

[2] P. B. Gilkey, Curvature and eigenvalues of the Laplacian for elliptic complexes, Adv. Math. 10 (1973) 344-382.

[3] M. F. Atiyah and I. M. Singer, The index of elliptic operators on compact manifolds, Bull. Amer. Math. Soc. 69 (1963) 422-433.

[4] M. Atiyah, R. Bott, and V. K. Patodi, On the heat equation and the index theorem, Invent. Math. 19 (1973) 279-330.

[5] V. A. Fock, The proper time in classical and quantum mechanics, Izv. Akad. Nauk. USSR (Phys.) 4-5 (1937) 551-568.

\footnotetext{
${ }^{7}$ see section 3.4 .3 of ref. [14]
} 
[6] J. Schwinger, On gauge invariance and vacuum polarization, Phys. Rev. 82 (1951) 664-679.

[7] B. S. DeWitt, Dynamical Theory of Groups and Fields, Gordon and Breach, New York (1965).

[8] B. S. DeWitt, Quantum theory of gravity. 1. the canonical theory, Phys. Rev. 160 (1967) $1113-1148$.

[9] B. S. DeWitt, Quantum theory of gravity. ii. the manifestly covariant theory, Phys. Rev. 162 (1967) 1195-1239.

[10] B. S. DeWitt, Quantum theory of gravity. iii. applications of the covariant theory, Phys. Rev. 162 (1967) 1239-1256.

[11] I. G. Avramidi, Heat kernel approach in quantum field theory, Nucl. Phys. Proc. Suppl. 104 (2002) 3-32.

[12] I. G. Avramidi, Heat Kernel and Quantum Gravity, Lecture Notes in Physics, Series Monographs, LNP:m64, Springer-Verlag, Berlin (2000).

[13] D. V. Vassilevich, Heat kernel expansion: user's manual, Phys. Rept. 388 (2003) 279-360 arXiv:hep-th/0306138.

[14] A. M. Charles and F. Larsen, Universal corrections to non-extremal black hole entropy in $\mathcal{N} \geq 2$ supergravity, JHEP 1506 (2015) 200 [arXiv:1505.01156].

[15] S. Banerjee, R. K. Gupta and A. Sen, Logarithmic Corrections to Extremal Black Hole Entropy from Quantum Entropy Function, JHEP 1103 (2011) 147 [arXiv:1005.3044].

[16] S. Banerjee, R. K. Gupta, I. Mandal and A. Sen, Logarithmic Corrections to $N=4$ and N=8 Black Hole Entropy: A One Loop Test of Quantum Gravity, JHEP 1111 (2011) 143 arXiv:1106.0080].

[17] A. Sen, Logarithmic Corrections to N=2 Black Hole Entropy: An Infrared Window into the Microstates, Gen. Rel. Grav. 44, no. 5, (2012) 1207-1266 [arXiv:1108.3842.

[18] A. Sen, Logarithmic Corrections to Rotating Extremal Black Hole Entropy in Four and Five Dimensions, Gen. Rel. Grav. 44 (2012) 1947-1991 arXiv:1109.3706].

[19] S. Bhattacharyya, B. Panda and A. Sen, Heat Kernel Expan sion and Extremal Kerr-Newmann Black Hole Entropy in Einstein-Maxwell Theory, JHEP 1208 (2012) 084 [arXiv:1204.4061.

[20] E. B. Manoukian, Rarita-Schwinger massless field in covariant and Coulomb-like gauges, Mod.Phys.Lett. A31 (2016) no.08, 1650047.

[21] A. L. Maroto and A. Mazumdar, Production of spin 3/2 particles from vacuum fluctuations, Phys.Rev.Lett. 84 (2000) 1655-1658 arXiv:hep-ph/9904206.

[22] H. P. Nilles, M. Peloso and L. Sorbo, Nonthermal production of gravitinos and inflatinos, Phys.Rev.Lett. 87 (2001) 051302 arXiv:hep-ph/0102264.

[23] R. Kallosh, L. Kofman, A. Linde and A. V. Proeyen, Gravitino production after inflation, Phys.Rev. D61 (2000) 103503 [arXiv:hep-th/9907124].

[24] E. S. Fradkin and A. A. Tseytlin, Off-shell One Loop Divergences in Gauged $O(N)$ Supergravities, Phys.Lett. 117B (1982) 303-308.

[25] E. S. Fradkin and A. A. Tseytlin, Quantum Properties of Higher Dimensional and Dimensionally Reduced Supersymmetric Theories, Nucl.Phys. B227 (1983) 252. 
[26] R. Endo, Heat kernel for spin 3/2 Rarita-Schwinger field in general covariant gauge, Class.Quant.Grav. 12 (1995) 1157-1164 [arXiv:hep-th/9407019].

[27] R. Seeley, Singular integrals and boundary value problems, Amer. J. Math. 88 (1966) 781809.

[28] R. Seeley, The resolvent of an elliptic boundary value problem, Amer. J. Math. 91 (1969) 889920.

[29] M. J. Duff, Observations on Conformal Anomalies, Nucl. Phys. B 125 (1977) 334.

[30] S. M. Christensen and M. J. Duff, New Gravitational Index Theorems And Supertheorems, Nucl. Phys. B 154 (1979) 301.

[31] S. M. Christensen and M. J. Duff, Quantizing Gravity With A Cosmological Constant, Nucl. Phys. B 170 (1980) 480.

[32] M. J. Duff and P. van Nieuwenhuizen, Quantum Inequivalence Of Different Field Representations, Phys. Lett. B 94 (1980) 179.

[33] N. D. Birrel and P. C. W. Davis, Quantum Fields in Curved Space, Cambridge University Press, New York (1982).

[34] P. B. Gilkey, Invariance theory, the heat equation and the Atiyah-Singer index theorem, Publish or Perish Inc., USA (1984).

[35] M. J. Duff and S. Ferrara, Generalized mirror symmetry and trace anomalies, Class. Quant. Grav. 28 (2011) 065005 [arXiv:1009.4439.

[36] P. B. Pal, Representation-independent manipulations with Dirac matrices and spinors (2007) arXiv:physics/0703214.

[37] G. De Berredo-Peixoto, A note on the heat kernel method applied to fermions, Mod.Phys.Lett. A16 (2001) 2463-2468 arXiv:hep-th/0108223.

[38] W. Rarita and J. Schwinger, On a Theory of Particles with Half-Integral Spin, Phys. Rev. 60 (1941) 61. 